\title{
Nanocrystalline Permanent Magnets with Enhanced Properties
}

\author{
M. LEONOWICZ* \\ Faculty of Materials Science and Engineering, Warsaw University of Technology \\ Wołoska 141, 02-507 Warszawa, Poland

\begin{abstract}
Parameters of permanent magnets result from the combination of intrinsic properties such as saturation magnetization, magnetic exchange, and magnetocrystalline energy, as well as microstructural parameters such as phase structure, grain size, and orientation. Reduction of the grain size into nanocrystalline regime $(\approx 50 \mathrm{~nm})$ leads to the enhanced remanence which derives from ferromagnetic exchange coupling between highly refined grains. In this study the fundamental phenomena, quantities, and structure parameters, which define nanophase permanent magnets are presented and discussed. The theoretical considerations are confronted with experimental data for nanocrystalline $\mathrm{Sm}-\mathrm{Fe}-\mathrm{N}$ type permanent magnets.
\end{abstract}

PACS numbers: 75.50.-y, 75.60.-d, 75.75.+a

\section{Introduction}

Nanocrystalline materials are built from the same atoms as their microcrystalline counterparts, however the crystallites have size less than $100 \mathrm{~nm}$. In fact, this critical size in real nanomaterials is much smaller. Nanocrystalline grains interact with electric field, domain walls and dislocations in different way than large crystallites. In this meaning nanocrystalline materials appear as an important class of new materials, which may become an issue for the next generations. Very fast development of nanomaterials has occurred within last 20 years. Among them are new tungsten carbide-cobalt wear resistant nanomaterials [1], partly crystalline soft magnetic nanomaterials [2], multilayer data storage materials [3], giant magnetoresistive [4] as well as catalytic [5] and structural nanomaterials [6]. Since 1990 nanomaterials have been implemented to the technology of permanent magnets. Modern permanent magnets are generally based on three systems: $\mathrm{Nd}-\mathrm{Fe}-\mathrm{B}[7,8]$,

*e-mail: mkl@inmat.pw.edu.pl 
$\mathrm{Sm}-\mathrm{Fe}-\mathrm{Co}[9]$ and $\mathrm{Sm}-\mathrm{Fe}-\mathrm{N}$ [10]. These magnets owe their excellent parameters to the combination of the intrinsic properties such as saturation magnetization, magnetic exchange and magnetocrystalline energy of the intermetallic phases, which form the material, and optimised microstructure and phase constitution. When the grains are small enough the material attains some proportion of magnetic anisotropy which leads to the enhanced remanence.

In this paper the basic definitions concerning permanent magnet nanomaterials are presented and confronted with experimental data for nanocrystalline $\mathrm{Sm}-\mathrm{Fe}-\mathrm{N}$ magnets.

\section{Why nanocrystalline structure?}

Polycrystalline materials are built of grains (crystallites), which are separated by grain boundaries. Each crystallite is a single crystal, whereas the boundary is a disordered area of enhanced energy. The smaller the grains the greater volume fraction of material is in a form of grain boundaries (Fig. 1). Because the material properties depend on the atom positions and interactions between them it is obvious that the properties of nanocrystalline body must differ substantially from its microcrystalline counterpart.

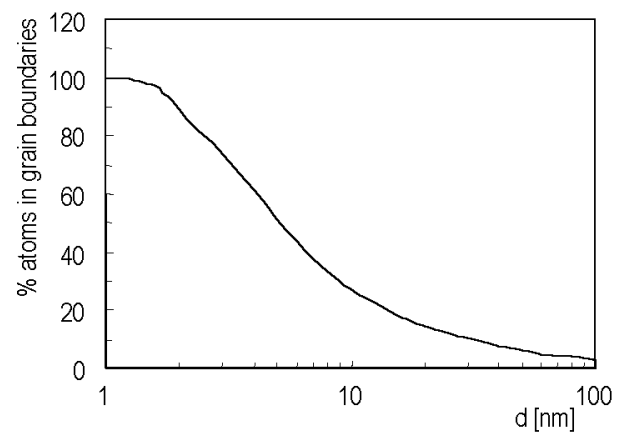

Fig. 1. Percentage of atoms in grain boundaries versus grain size. Boundary thickness $1 \mathrm{~nm}$.

The magnetic properties of permanent magnets are characterised by the parameters of hysteresis loop: coercivity $(\mathrm{Hc})$, remanence $(\mathrm{Br})$ and maximum energy product $\left((B H)_{\max }\right)$ (Fig. 2$)$. The greater the values of those parameters the better the magnet. High coercivity results from high magnetocrystalline anisotropy of intermetallic phases in the magnet and can be further increased by formation of a paramagnetic phase in the grain boundaries, which isolate them magnetically. Modern permanent magnets generally exhibit high coercivity, thus improvement of the properties should be made by enhancement of the remanence. The usable $(B H)_{\max }$ are always much smaller that the theoretical value expressed by the formula 


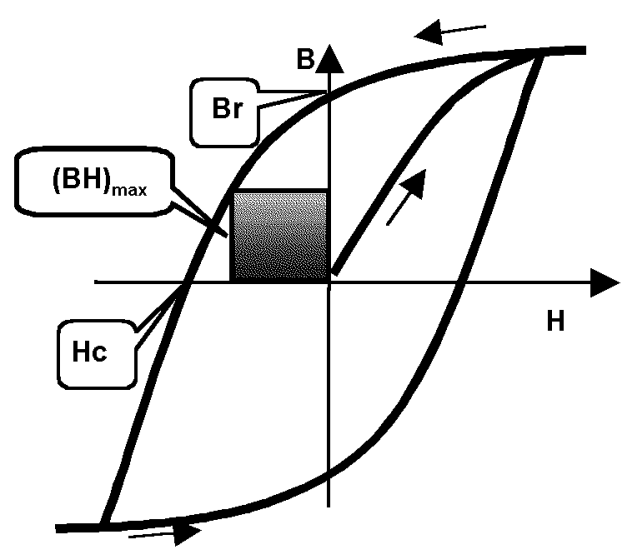

Fig. 2. Hysteresis loop of a permanent magnet.

$$
(B H)_{\max } \leq \frac{\mu_{0} M_{\mathrm{r}}^{2}}{4} \leq \frac{\mu_{0} M_{\mathrm{s}}^{2}}{4} .
$$

According to the Stoner-Wohlfart prediction [11] the remanence $M_{\mathrm{r}}$ of polycrystalline material being a mixture of single domain, non-interacting magnetically particles is equal to the half of the saturation magnetization $M_{\mathrm{s}}$

$$
M_{\mathrm{r}}=\frac{M_{\mathrm{s}}}{2}
$$

However, the remanence of nanocrystalline magnets appears to be substantially enhanced above this value (Fig. 3). This can be achieved by processing of magnets, which do not have paramagnetic, isolating phase in grain boundaries and have grain size less than $50 \mathrm{~nm}$. The enhanced remanence is due to the strong exchange coupling of magnetic moments in adjacent nanograins. Minimization of the exchange energy tends magnetic moments in the material to align parallel to each other whereas the anisotropy energy becomes minimum when the magnetic moments are parallel to the crystallographic easy magnetization direction in
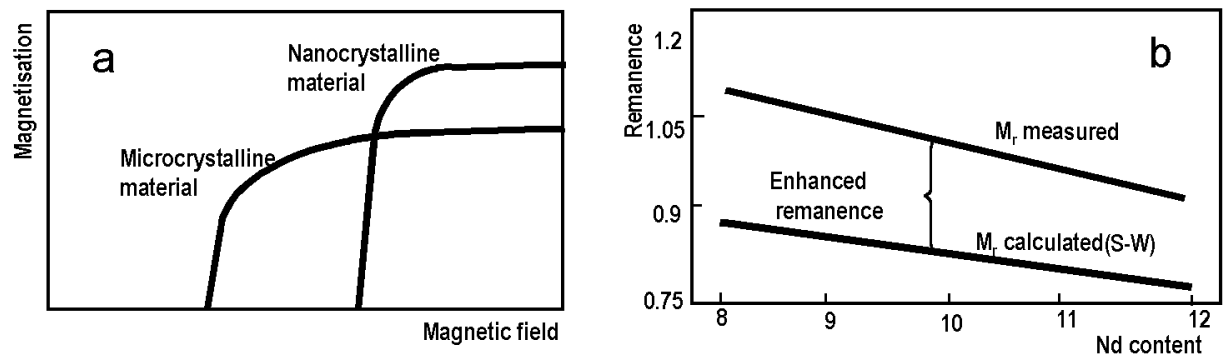

Fig. 3. (a) Parts of the hysteresis loops for microcrystalline and nanocrystalline magnets. (b) Remanence of nanocrystalline magnets versus Nd content. 
each grain. When grains are small enough the exchange length $L$ from crystallites, oriented favourably with their easy magnetization axes to the external magnetic field, covers substantial parts of the neighbouring grains. The exchange length is proportional to the exchange constant $A$ and reversibly proportional to average anisotropy constant $\langle K\rangle$ :

$$
l=\left(\frac{A}{\langle K\rangle}\right)^{\frac{1}{2}}, \quad\langle K\rangle=\frac{K_{1}^{4} d_{z}^{6}}{A^{3}} .
$$

The latter coefficient strongly depends on the grain size $d_{z}$. Thus, in nanocrystalline magnets the exchange length covers substantial volume of neighbouring crystallites. As a result the material gains some proportion of magnetic anisotropy in a crystallographically isotropic state (Fig. 4).

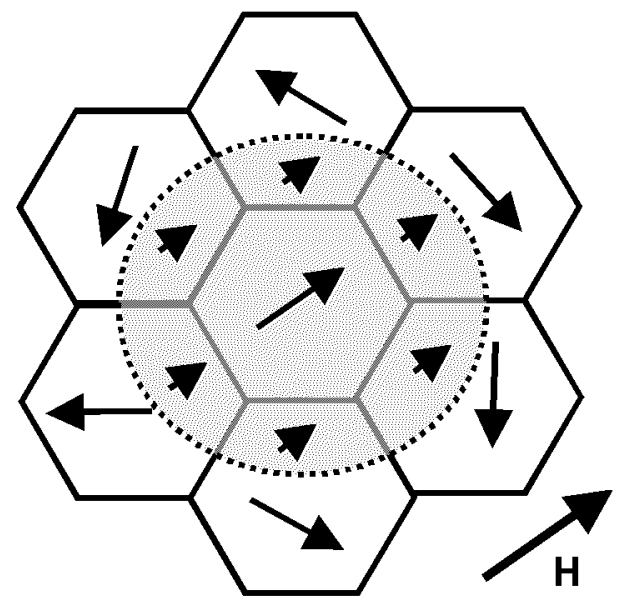

Fig. 4. Scheme of the magnetic exchange interactions in nanocrystalline $\mathrm{Nd}-\mathrm{Fe}-\mathrm{B}$ magnets.

The remanence can be further increased when the material contains some proportion of iron. Usually, in microcrystalline material, soft magnetic phase acts as a microstructural defect, which is a source of demagnetizing field. The hysteresis loop of such a material has poor squareness and thus low $(B H)_{\max }$ (Fig. 5). However, a nanocrystalline magnet, which contains some proportion of nanocrystalline iron, gains macroscopically magnetic properties characteristic of a single-phase magnet. Such magnets are called magnetic nanocomposites. They combine high uniaxial magnetocrystalline anisotropy of hard magnetic phases, $\mathrm{Nd}_{2} \mathrm{Fe}_{14} \mathrm{~B}$ or $\mathrm{Sm}_{2} \mathrm{Fe}_{17} \mathrm{~N}_{3}$, with high saturation magnetization of iron $\left(\mathrm{Nd}_{2} \mathrm{Fe}_{14} \mathrm{~B}-M_{\mathrm{s}}=1.6 \mathrm{~T}\right.$; $\mathrm{Fe}-M_{\mathrm{s}}=2.2 \mathrm{~T}$ ). The magnetic moments of Fe follow the moments of the hard magnetic phase and the remanence is a combination of the enhancement resulting from the nanocrystalline structure and high magnetic moment of Fe. Summarising this paragraph we can distinguish: (i) microcrystalline high coercivity and low 


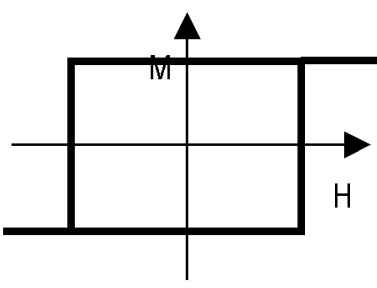

Hard magnetic

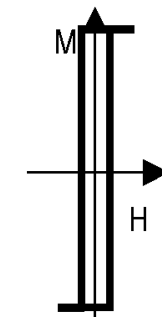

Soft magetic

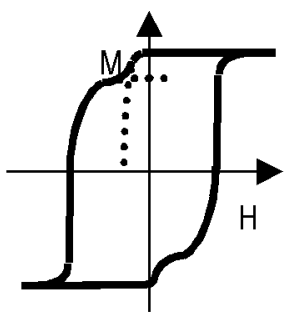

Microcrystalline

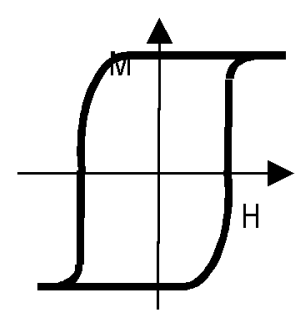

Nanocomposite

Fig. 5. Schematic hysteresis loops for the hard magnetic material, soft magnetic material, microcrystalline mixture of hard and soft materials and a nanocomposite magnet.

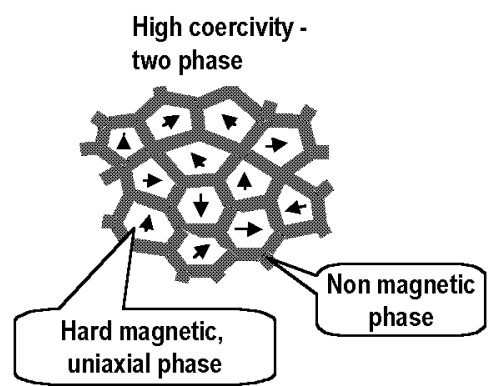

Exchange decoupled

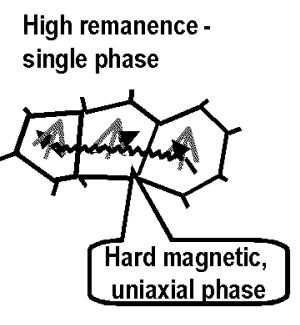

Exchange coupled
High remanence -

composite

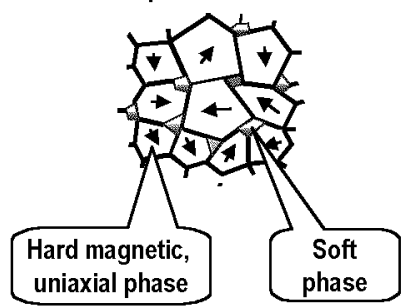

Exchange coupled

Fig. 6. Scheme of the: high coercivity, two-phase magnet; single phase, exchange coupled, nanocrystalline magnet; exchange coupled nanocomposite magnet.

remanence magnets, having large grains, which are separated magnetically by a paramagnetic phase (typical of sintered $\mathrm{Nd}-\mathrm{Fe}-\mathrm{B}$ magnets), (ii) nanocrystalline, single phase magnets with enhanced remanence, which often are called "spring magnets" because the magnetic moments tend to be aligned parallel as they were connected mechanically by springs and (iii) nanocomposite two-phase magnets (Fig. 6).

\section{Formation of nanostructure in $\mathrm{Sm}-\mathrm{Fe}-\mathrm{N}$ magnets}

Nanocrystalline and nanocomposite magnets are generally processed using either rapid solidification (RS) or mechanical alloying (MA). Whereas in the former method the material is obtained directly from melt, for the latter the hard magnetic phase is formed in the course of annealing. The effects of annealing strongly depend on the structure of the as-milled powder. Mechanical alloying is based on high-energy ball milling of elementary powders (Fig. 7). In the course of extensive milling powder particles are repeatably crushed and cold-welded. Finally, depending on the energetical relations between different phases, either nanocrystalline or 


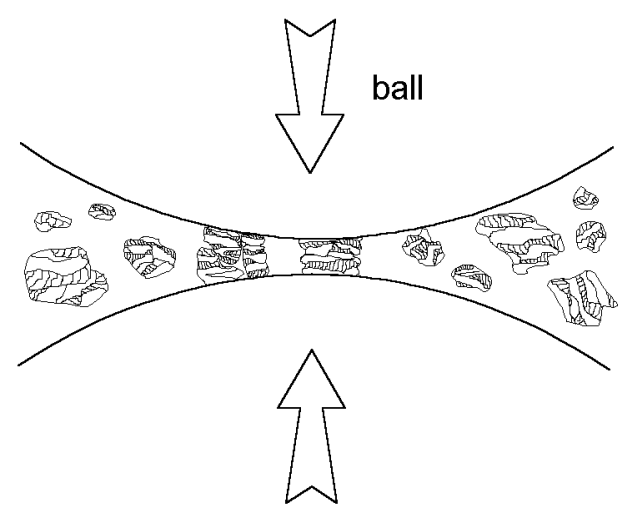

Fig. 7. Scheme of mechanical alloying.

amorphous structure is formed. The following annealing results in crystallization of the amorphous phase and formation of the nanocrystalline structure.

In this study nanocrystalline $\mathrm{Sm}$-Fe powders were processed with the use of mechanical alloying of elementary Sm and Fe powders. The milling was followed by diffusion annealing at temperatures $650-800^{\circ} \mathrm{C}$ within 1 hour. Annealed powders were subsequently nitrided in pure nitrogen gas at a temperature $460^{\circ} \mathrm{C}$ within $3 \mathrm{~h}$.

$\mathrm{Sm}-\mathrm{Fe}-\mathrm{N}$ type permanent magnets have been extensively studied since their discovery by Coey and Sun in 1990 [12]. The coercivity, remanence and maximum energy product depend on the microstructure, which is formed in the course of the processing. The magnets can be either single phase $\left(\mathrm{Sm}_{2} \mathrm{Fe}_{17} \mathrm{~N}_{3}\right.$ often with some excess of $\mathrm{Sm}$ ) or two phase nanocomposite $\left(\mathrm{Sm}_{2} \mathrm{Fe}_{17} \mathrm{~N}_{3}-\alpha\right.$ - $\left.\mathrm{Fe}\right)$. Reduction of the grain size into nanocrystalline regime $(\approx 50 \mathrm{~nm})$ leads to the enhanced remanence which derives from ferromagnetic exchange coupling between highly refined grains and from the presence of the $\alpha-\mathrm{Fe}$. The processing of Sm-Fe-N magnets comprises mechanical alloying, diffusion annealing and nitriding. Mechanical alloying led to the formation of microstructure, which consisted of nanoscale $\alpha$-Fe crystals, which were embedded in amorphous Sm-rich matrix. The minimum milling time which allowed producing hard magnetic properties was $50 \mathrm{~h}$ in our experiment (Fig. 8a). Further extension of milling time did not affect the properties.

Diffusion annealing produced nanocrystalline material (mean crystallite size $-50 \mathrm{~nm}$ ) which, depending on the composition, consisted of the combination of the following phases: $\mathrm{Sm}_{2} \mathrm{Fe}_{17}, \alpha-\mathrm{Fe}$ and $\mathrm{Sm}$. The optimal annealing temperature depended on the alloy composition and was higher for alloys, which contained more samarium (Fig. 8b). In the course of annealing, crystallization and grain growth of the $\mathrm{Sm}_{2} \mathrm{Fe}_{17}$ phase occurs. Maximum properties were obtained after about 20-30 min annealing. Further extension of the annealing time led to deterioration of ${ }_{J} H_{c}$, which was accompanied by some increase in $J_{\mathrm{r}}$. 

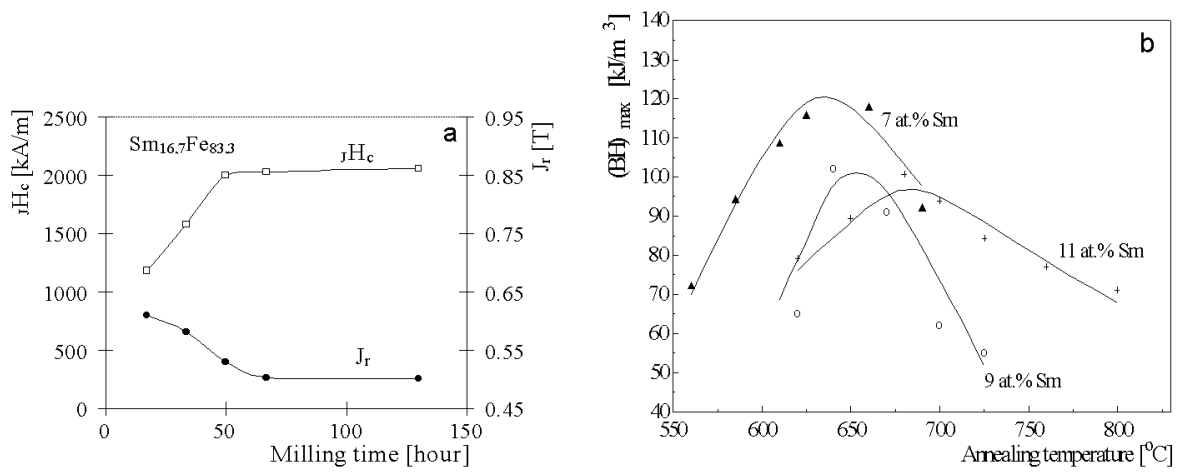

Fig. 8. (a) Effect of milling time on the coercivity of Sm-Fe alloy (after complete processing) and (b) of annealing temperature on the maximum energy product of $\mathrm{Sm}-\mathrm{Fe}$ alloys having different compositions (after complete processing).

Nitriding leads to the formation of a solid solution of nitrogen on a basis of the $\mathrm{Sm}_{2} \mathrm{Fe}_{17}$ intermetallic phase. The powders were nitrided at $460^{\circ} \mathrm{C}$ for $3 \mathrm{~h}$. The nitriding dramatically changes the magnetic properties (Fig. 9). The magnetic anisotropy grows from $2.5 \mathrm{~T}$ (planar) to $14 \mathrm{~T}$ (axial), saturation magnetization grows from $1 \mathrm{~T}$ to $1.54 \mathrm{~T}$ and Curie temperature from $120^{\circ} \mathrm{C}$ to $470^{\circ} \mathrm{C}$.

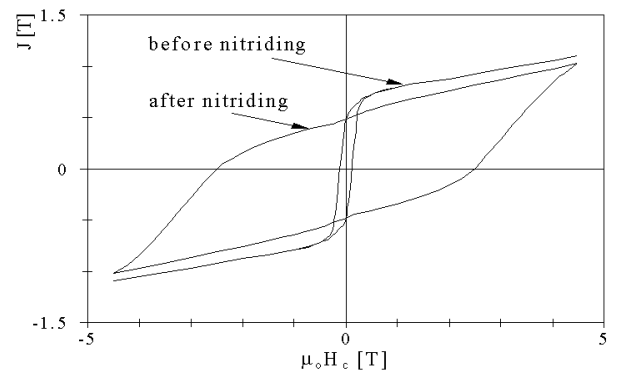

Fig. 9. Hysteresis loops for $\mathrm{Sm}-\mathrm{Fe}-\mathrm{N}$ alloy before and after nitriding.

The magnetic properties for alloys having different Sm contents after complete processing are shown in Fig. 10. The highest coercivity of $2008 \mathrm{kA} / \mathrm{m}$ was achieved for nitrided $\mathrm{Sm}_{16,7} \mathrm{Fe}_{83,3}$ alloy. $J_{\mathrm{r}}$ increased with increasing Fe content. It is interesting to note that the stoichiometric alloy $(10.5 \% \mathrm{Sm})$ had $J_{\mathrm{r}}=0.9 \mathrm{~T}$ which was higher than the expected for non-interacting particles value of $0.75 \mathrm{~T}$ and resulted from nanocrystalline structure. The highest $(B H)_{\max }$ of $175 \mathrm{~kJ} / \mathrm{m}^{3}$ was achieved for the alloy having $7 \mathrm{at} \% \mathrm{Sm}$.

Increase in the $\mathrm{Sm}$ concentration resulted in a change of phase constitution and thus the magnetic properties of magnets. The diagram showing phase structure versus composition for $\mathrm{Sm}-\mathrm{Fe}-\mathrm{N}$ magnets and its effect on the magnetic properties is schematically shown in Fig. 11. 

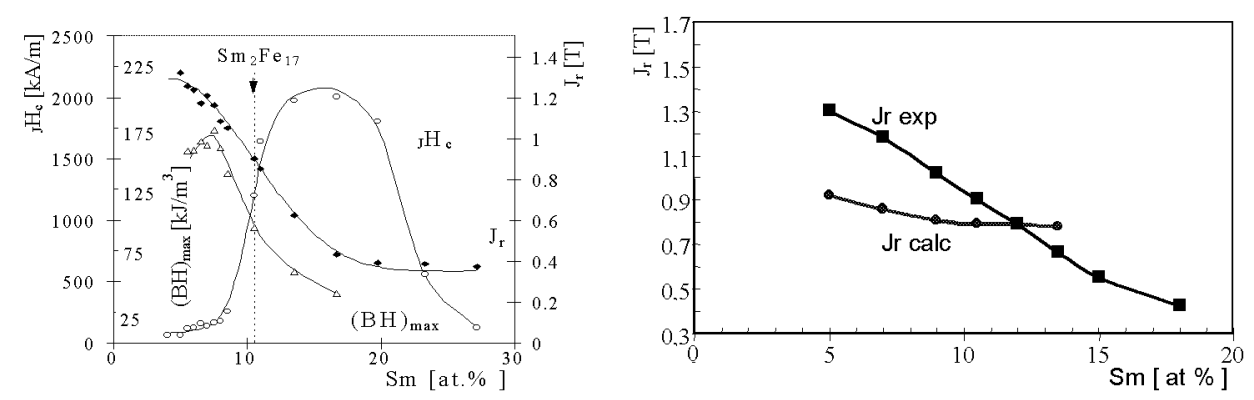

Fig. 10. Effect of $\mathrm{Sm}$ content on the magnetic properties of nitrided $\mathrm{Sm}_{x} \mathrm{Fe}_{1-x}$ alloys, and comparison of the experimental and calculated values of the $J_{\mathrm{r}}$.

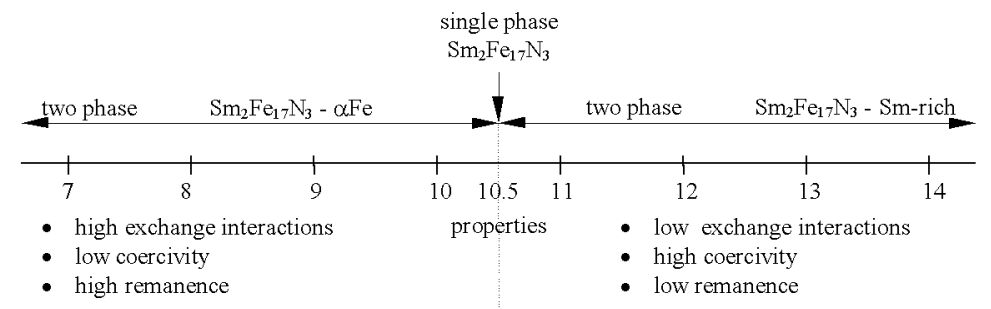

Fig. 11. Phase structure versus composition for $\mathrm{Sm}-\mathrm{Fe}-\mathrm{N}$ magnets and its effect on the magnetic properties.

\section{Further developments}

Further developments of nanocrystalline magnets should consider processing of anisotropic composite magnets. Such a structure would comprise hard magnetic uniaxial nanocrystalline matrix, which would contain small inclusions of iron or iron-cobalt (Fig. 12). Analytical calculations of the $(B H)_{\max }$ for such an anisotropic magnet, composed from $2.5 \mathrm{~nm}$ crystallites of $\mathrm{Sm}_{2} \mathrm{Fe}_{17} \mathrm{~N}_{3}$ phase and $9 \mathrm{~nm}$ crystallites of $\mathrm{Fe}_{65} \mathrm{Co}_{35}$ would give theoretical $(B H)_{\max }=1 \mathrm{MJ} / \mathrm{m}^{3}$ [13]. Currently best anisotropic magnets exhibit $(B H)_{\max }=0.4 \mathrm{MJ} / \mathrm{m}^{3}$.

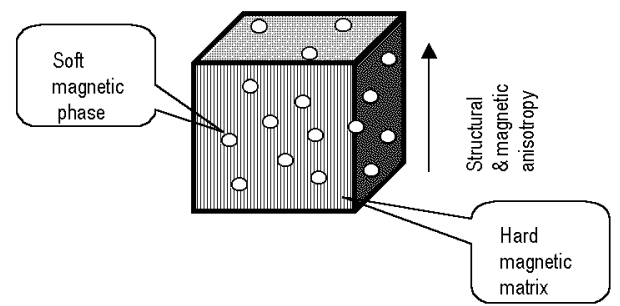

Fig. 12. Model of anisotropic nanocomposite magnet. 


\section{References}

[1] B.H. Kear, L.E. McCandlish, Nanostruct. Mater. 3, 19 (1993).

[2] Y. Yoshizawa, S. Oguma, Y. Yamaguchi, J. Appl. Phys. 64, 6044 (1988).

[3] H.J. de Witt, C.H.M. Wittmer, F.W.A. Dirne, Adv. Mater. 3, 356 (1991).

[4] J.Q. Xiao, J.S. Jiang, C.L. Chien, Phys. Rev. Lett. 68, 3749 (1992).

[5] D.D. Beck, R.W. Siegel, J. Mater. Res. 7, 2840 (1992).

[6] K. Nihara, J. Ceram. Soc. Jap. 99, 974 (1991).

[7] A. Manaf, R.A. Buckley, H.A. Davies, M. Leonowicz, J. Magn. Magn. Mater. 101, 360 (1991).

[8] H.A. Davies, A. Manaf, M. Leonowicz, Z.P.Z. Zhang, S.J. Dobson, R.A. Buckley, Nanostruct. Mater. 2, 197 (1993).

[9] H. Nagel, A. Menth, IEEE Trans. Magn. 14, 53 (1978).

[10] D. Januszewski, M. Leonowicz, in: Proc. 4th Int. Conf. Achievements in Mech. Mater. Eng., Wista (Poland) 1996, Ed. L.A. Dobrzeński, Wyd. Politechniki Śląskiej, Gliwice, p. 139.

[11] E.C. Stoner, W.P. Wohlfarth, Philos. Trans. R. Soc. Lond. A 240, 599 (1948).

[12] J.M.D. Coey, H. Sun, J. Magn. Magn. Mater. 87, L251 (1990).

[13] R. Skomski, J. Appl. Phys. 76, 7059 (1994). 